\title{
Challenges for reducing food waste
}

\author{
Alexandra-Catalina CHINIE \\ Bucharest University of Economic Studies, Bucharest, Romania \\ catalina.chinie@fabiz.ase.ro
}

\begin{abstract}
According to the Food and Agriculture Organization of the United Nations, approximately one third of the food produced globally is wasted, by the private sector and consumers. Reducing food waste would have an essential role in fighting world hunger, by redistributing food that is not consumed. However, achieving this goal would not only have a positive social impact, but also an economic and environmental impact. The subject of food waste is strongly tied to the objectives of several international organizations and of public institutions that have lead awareness campaigns and have developed goals and policies for the reduction of the phenomenon. Reducing food waste would contribute to the achievement of the 12th Sustainable Development Goal of the United Nations, "Ensure sustainable consumption and production patterns". For an efficient strategy, it is necessary that organizations and the involved people analyze and eliminate research gaps and the barriers for the reduction of food waste, both among consumers and private companies, such as producers, distributors, retailers, as well as the food service industry. The present article reviews the main challenges and barriers in achieving the reduction of food waste, both in the private sector, and among consumers. Among the main challenges that need to be tackled are the quantification of wasted food along the value chain, systematic reporting of wasted food and the evaluation of the causes of food waste.
\end{abstract}

Keywords: food waste, challenges, food waste causes, food waste quantification.

\section{Introduction}

Reducing food loss and waste is an objective that has been put on the agenda of individual states and international organizations. In 2015, the United States Department of Agriculture and the United States Environmental Protection Agency have announced that they plan to reduce food loss and waste by half by 2030 (EPA, 2020). Reducing food waste is correlated with the 2nd and the 12th Sustainable Development Goals of the United Nations - "Zero Hunger" and "Responsible Consumption and Production". The European Union is also committed to reducing food waste by half until 2030, and as part of its strategy in order to do so, it has established the EU Platform on Food Losses and Food Waste in 2016, where prevention measures can be defined, best practices can be shared and progress can be evaluated over time (European Commission, 2020).

Waste is generated along the entire food chain (Morone et al., 2019). Identifying ways of fighting food waste requires a good knowledge of why food is wasted along its entire value chain. On one hand, there might be poor business decisions or processes that lead to food being wasted (Bratianu, 2015; Teller et al., 2018; Mena et al., 2011), while on the other hand, there are consumers that have an impact on the phenomenon due to several behavioral aspects, among which, refusing to buy food that doesn't look optimal anymore (Cicatiello et al., 2016), refusing to buy food that is approaching the ,best before” date, as well as buying more food that needed and not consuming it in time (Papargyropoulou et al., 2014). According FAO (Gustavsson et al., 2011) and previous research (Ju et al., 2017; Bio Intelligence Service, 2010; Parfitt et al., 2010), food is wasted in the earlier stages of the supply chain in the case of developing countries, and in the later stages of the supply chain, in the case of developed countries. 
As prevention measures are regarded as the most efficient way to combat food waste (Papargyropoulou et al., 2014), several important aspects must be considered - what triggers food waste along the food chain and which are the most efficient measures and instruments that are currently being used to reduce it. In order to assess the efficiency of food waste reduction actions, the food being thrown away must also be quantified. Quantification efforts can exceed measuring the weight of wasted food and may also refer to the value of the wasted food. In each of these aspects there are barriers to overcome, which have become the subject of a growing body of research in the last years.

The scope of this article is to present a systematic review of the main challenges and barriers to reducing food waste. Future research of the author is determined by the results of this study.

\section{Literature review}

\section{Causes of food waste}

Research shows that essentially, people are concerned about food waste (Graham-Rowe et al., 2014). A study on food waste has shown that the intention to reduce food waste isn't significantly correlated with a reduced self-reported food waste (Stefan et al., 2013), although other researchers have had contradicting results (Graham-Rowe et al., 2015). According to a study performed by the European Commission, the main causes of food waste are found both at business and consumer levels and include portion size, labelling, packaging and storage issues, as well as awareness, preferences, planning and socio-economic factors (Bio Intelligence Service, 2010). However, analyses regarding the drivers of food waste are scattered (Schanes et al., 2018).

People's approach to food waste represents a growing body of literature in the consumer behavior and environmental psychology area (Visschers et al., 2016), as well as within the social practice theory (Hebrok and Heidenstrøm, 2019; Romani et al., 2018). A systematic review of research concerning the consumer food waste was performed by Schanes et al. (2018). The authors synthetized food waste reasons into two main categories: understandings and perceptions of food waste, and food-related household practices and routines - planning, shopping, storage, cooking, eating, managing leftovers, assessing edibility and disposal (Schanes et al., 2018). Results of Leverenz et al. (2019) are in line with those of Schanes et al. (2018) - the authors have identified the following drivers for household food waste: "cooking too much, spoiling during storage, not using saved leftovers in time, expired shelf life, mistakes in handling and other reasons" (Leverenz et al., 2019). Situational factors also come into play when discussing household food waste. The household size, having children and spending more money on groceries have a negative effect on food waste (Parizeau, 2015). On the other hand, shopping groceries more frequently can lead to a reduced food waste (Williams et al., 2012).

Results of the study of barriers to reducing food waste, performed by Graham-Rowe et al. (2015), have shown that psychological factors such as being a good provider and offering the family healthy and/or ample food, the desire to minimize inconvenience and a lack of awareness and information may represent barriers to the reduction of food waste. On the other hand, Hebrok and Heidenstrøm (2019) argue that informing consumers about the societal aspects of food waste is not enough to help reduce the phenomenon. Instead, information and awareness campaigns should highlight the effects of everyday practices (Hebrok and Heidenstrøm, 2019). In this regard, the authors underline the importance of an unstructured approach to food waste, such as the fridge analysis, in providing a more detailed context of the causes of the phenomenon 
(Hebrok and Heidenstrøm, 2019). Leverenz et al. (2019) has shown that receiving coaching for food waste reduction managed to reduce the quantity of wasted food significantly, with the majority of the households included in the research reducing the quantity of wasted food by more than a half.

In a study performed in UK and Ireland, Mena et al. (2011) have classified the causes for food waste at the supplier-retailer interface in three categories: (1) megatrends such as increasing demand for fresh products, products that are out of season, and those that contain no preservatives, (2) natural constraints and (3) management root causes. Opportunities to reduce food waste at this stage of the supply chain lie with removing the challenges at management level (Mena et al., 2011) and require high management commitment (Filimonau and Gherbi, 2017). Cicatiello et al. (2016) listed the following causes of food waste at the retailer level: damaged packaging, incorrect storage, unsuitable quality standards, blemished/misshapen products and unpurchased products, while fresh produce, decomposition and expiration of packaged goods were considered factors for food loss. In the food service sector, over-preparation and incorrect storage were listed as food waste factors, while consumer leftovers and food preparation waste were listed as food loss causes (Cicatiello, 2016).

The research of Teller et al. (2018) underlined the interdependence between the increasing customer expectations and higher retailers' standards for product and service quality. Results of their research also highlighted that hypermarkets and supermarkets have a higher potential for the reduction of food waste (Teller et al., 2018).

Based on the Organizational Theory, Özbük and Coșkun (2020) have classified factors affecting food waste generated within the downstream segment of the supply chain into internal, micro-environmental and macro-environmental factors. Among internal factors the authors have listed organizational, operational, marketing-related, product-related and food waste handling practices (Özbük and Coșkun, 2020).

In order to take efficient measures of reducing food waste, a holistic approach regarding the drivers of food waste must be considered. Considering the supply chain, causes related to food waste must be viewed from an integrated approach - decisions that are taken within a segment of the supply chain, at retailer level for example, can have an influence of food being wasted at consumer level (Aschemann-Witzel et al., 2017). Such an example is outlined in the work of Lee (2018), who studied the correlation between food retail and the amount of food wasted in Seoul households. Results showed that consumer tend to purchase more than they need due to bulk packaging and buying promotions (Bravi et al., 2020; Lee, 2018), as well as travel time and frequency of buying (Lee, 2018). Schanes et al. (2018) also underline the need of frameworks that are aimed farther than an individual level, and take a more holistic approach, empowering actors across the supply chain (Schanes, 2018). Studies have concentrated more on individual actors of the supply chain and rarely on several parties (Özbük and Coșkun, 2020).

\section{Quantifying the amount of wasted food}

In order to correctly assess the phenomenon of food waste, develop effective strategies and track the progress of corrective actions, food loss and waste must be measured as accurately as possible. In this way, most efficient measures or tools that help mitigate this issue can be identified. Consequently, a growing body of literature has tried to tackle this matter (Elimelech et al., 2019; Elimelech et al., 2018; Eriksson et al., 2019; Caldeira et al., 2019; Eriksson et al., 2018a; Ju et al., 2017). According to Gustavsson et al. (2011), approximately one third of food is lost. However, calculation efforts so far provide a "narrow temporal, geographical, and food 
supply chain coverage" (Xue et al., 2017). According to Corrado and Sala (2018), estimates for food waste range from 194 to $389 \mathrm{~kg} / \mathrm{p} / \mathrm{y}$ globally and from $158 \mathrm{tp} 290 \mathrm{~kg} / \mathrm{p} / \mathrm{y}$ in the European Union.

For reliable aggregated data, as well as for comparison means, a common reporting standard must be used (Eriksson et al., 2018b). The Food Loss and Waste Accounting Standard provides ,accounting and reporting requirements that can be used consistently by entities around the world", while also offering universally applicable definitions for food loss and waste (FLW Protocol, 2016). However, even when applying reporting standards for the quantification of food waste, certain limitation must be considered. The FLW Protocol mentions 12 sources of uncertainty for food waste and loss evaluation, among which: systematic errors (bias), methodological errors, data-processing errors, converting amounts to weight, assumptions, uncertainty in data used for inference based methods, model uncertainty and third-party data (FLW Protocol, 2016).

According to Beretta et al. (2013), the overall quantity of food that is wasted or lost does not provide sufficient information in order to mitigate the negative effects, and should be accompanied by information such as what type of food is wasted and where in the supply chain food that is wasted at the later stages of supply chain had also required transportation, storage, packaging and processing, as opposed to food that was wasted in the first stage of the supply chain.

Several researchers have studied the generation of food waste at each stage of the supply chain, using the mass flow analysis (Caldeira et al., 2019; Beretta et al., 2013), or aggregating data at one of the supply chain stages (Buzby et al., 2015). Most of the research aimed at quantifying food waste generation across the supply chain has relied on secondary data (Xue et al., 2017). Following their work on quantifying food waste and loss, Caldeira et al. (2019) have underlined the need for more primary data on waste generated along the supply chain.

Analyzing the results of food waste assessments carried out by the Bio Intelligence Service on behalf of the European Commission (Bio Intelligence Service, 2010) and by the Swedish Institute of Food and Biotechnology on behalf of FAO (Gustavsson et al., 2011), Bräutigam et al. (2014) concluded that these lack comparability. Reasons for this would be using different sources for the estimations, different data collection methods and extrapolating results from other analyses at a different regional level (Bräutigam et al., 2014). Another issue was the lack of a common definition for "food waste" and "food loss" (Bräutigam et al., 2014), which the FLW Protocol tried to solve by offering a universally applicable definition (FLW Protocol, 2016). The EU-Fusions report on European food waste levels tried to tackle these issues by using only data from comparable methodologies (Stenmarck et al., 2016). Results of the FUSIONS project showed that at the EU-28 level, $20 \%$ of food is wasted, with the highest percentage being generated at within households and the processing sector $-72 \%$ in total (Stenmarck et al., 2016).

Food waste at household level can be assessed through several methods, and can be measured by poundage, calories or money value loss (Gallo, 1980). On one hand, the amount of wasted food can be measured by weight (WRAP, 2020) or tracked in a diary (Leverenz et al., 2019; Williams et al., 2012; Gallo, 1980) - these methods however, can influence consumers to waste less, due to increased awareness. Researcher Anthony E. Gallo mentioned another three methods in his 1980 paper on consumer food waste (Gallo, 1980): the archeological method foresees that the food thrown away is analyzed from the household garbage; the inferential method is based on the calculation of the difference between the amount of food bought, and the 
amount of food consumed - the result being the amount of wasted food; plate examination, where the unconsumed portion of food was subtracted from the portion of food served (Gallo, 1980).

Most research that wanted to quantify food waste have used self-reported data for their study (Leverenz et al., 2019; Romani et al., 2018; Visschers et al., 2016). This method, however, could lead to a quantification gap between what food loss and waste was actually generated and what quantities have been self-reported.

PICBE | 823

With regard to food waste quantification, different issues arise for each step in the value chain. Parfitt et al. (2010) mention national and regional legislation, accounting methodologies, corporate policies and managerial practices as issues for the estimation of produced food waste. Eriksson et al. (2012) showed that siginificant quantities of food waste remains unrecorded. For the food services sector, Eriksson et al. (2018a) mention short and infrequent quantification times, as well as large methodological variations as limitations. In this respect, the authors have proposed a framework for food waste quantification, which aims at making the process more efficient and allowing for comparability between different catering units; however, as mentioned by the authors, the full potential of the framework could be reached by using electronic food waste quantification (Eriksson et al., 2018a).

A research performed by Cicatiello and Franco (2020) has studied the difference between the reported amount of wasted food using the standard reporting procedure in place within retail stores, and an improved method of measurement, aimed at collecting the real quantities of wasted food. The results of the study has shown that the difference between the two types of measurement is significant (Cicatiello and Franco, 2020), thus underlining the difficulty of correctly quantifying the amount of food that is wasted.

\section{Further challenges for the reduction of food waste}

Apart from requirements to further analyze the causes of food waste along the supply chain, as well as to establish robust quantification methods of the food waste generation, that can be used for different units, at different stages of the supply chain, and the need to evaluate food waste on a periodical basis, there are several other aspects that also must be tackled. The need to research the impact of economic and regulatory measure on food waste was mentioned by Priefer et al. (2016) in their paper on food waste prevention in Europe. Correlated with this aspect, ReFED (2020) includes the assessment of ,macro-level economic changes resulting from food waste reductions", as an opportunity for nonprofit organisations and academic members.

According to Morone et al. (2019), a better connection between social scientists, economists, IT and software engineers and designers, and physical scientists is required for the development of integrated strategies for food waste reduction.

The research of Gokarn and Kuthambalayan (2017) has identified 33 challenges to reducing food waste in this segment of the supply chain, which were grouped in 9 categories food characteristics, supply chain uncertainty, information technology, supply chain partnerships, operational capability, consumer behavior, market infrastructure, food policy and regulation and supply chain networks. Out of these groups, the authors have identified food characteristics, supply chain uncertainty, food policy and regulations and market infrastructure to be the main barriers to food waste reduction for Indian agri-food companies (Gokarn and Kuthambalayan, 2017).

\section{Methodology}

A review of the current body of knowledge regarding barriers and challenges to food waste was performed. In order to identify research gaps and challenges to reducing food waste, a search of 
peer-reviewed journal articles published in English was performed. Firstly, a review of articles found searching for "food waste challenges" and "food waste reduction barriers" was performed. Based on results of this analysis, a further search was performed using the following key words: "food waste calculation", "consumer behavior", "food waste" and "food waste awareness". Papers that were based on single case studies, such as the study of a unit, were mainly excluded from the review. Papers that focus on the waste of a single product were also excluded from the study. By reading the abstracts, further papers were excluded from the review, such as those focused on food science. Further barriers and challenges to reducing food waste were derived from the analysis of the selected papers. The scientific databases used for the search were Web of Science, Scopus and Science Direct.

\section{Results and discussions}

The literature review reveals the most relevant aspects that must be tackled in order to reduce food waste, through efficient strategies at micro and macro level, as well as a correct assessment of the phenomenon.

A meta-analysis of previous researches reveals that the drivers of food waste need to be studied and handled from an integrated approach, taking into consideration the interdependencies between different actors across the supply chain (Bravi et al., 2020; Aschemann-Witzel et al., 2017; Lee, 2018; Cicatiello et al., 2017). Such an example would be raising awareness among consumers and organizing campaigns in order to change the perception of consumers towards products that don't look attractive, but are edible. This way, food waste is reduced at retailer level, but also at producer or processing level, given that the standards of retailers for purchased goods also change and allow also for "unattractive" products to be purchased. At the same time, reconsidering the sale of bulk packaging and promotions at retailer level can reduce food waste among consumers (Bravi et al., 2020; Lee, 2018).

Contextual, unstructured approaches for the analysis of food waste generation have proven to offer relevant information into the matter (Hebrok and Heidenstrøm, 2019). Further similar analyses are required in order to provide a complete root-cause analysis that can effectively support the formulation of policies and support instruments that fight against food waste generation.

For governments and organizations that lead awareness campaigns for food waste prevention, it is important to also include specific information regarding everyday practices that can help people reduce the amount of food that they waste (Hebrok and Heidenstrøm, 2019).

Another issue that needs to be tackled for a better management of the phenomenon is food waste quantification. Removing uncertainties and errors of food waste quantification can support the identification of the most efficient policies or strategies for food waste reduction. For the results to be comparable, common reporting standards must be used. The European Commission has published the EU Decision laying down a format for reporting of data on food waste and for submission of the quality check report (EU Decision 2019/2000). Member states of the European Union will collect data on food waste starting with 2020 and will report this information by mid2022. Aggregate information on food waste based on primary data can offer a relevant insight for policy-makers and organizations that are working to reduce food waste.

In order to overcome issues regarding the quantification of food waste, more attention and research efforts must be focused on technologies that can help measure the amount and type of wasted food, while ensuring convenience. An example of such technology is the artificial intelligence enabled scale that identifies and weighs food that is thrown away (Winnow Solution, 
2020). Moreover, artificial intelligence can reduce food waste by improving stock management (Kor et al., 2017) and adjusting prices according to the freshness of the products (Deloitte, 2018).

\section{Conclusion}

Reducing food waste is a Sustainable Development Goal that has attracted the implication of governments, organizations, private companies and the general public. Although actions aimed at mitigating this phenomenon have increased, there are still barriers to overcome in order to reduce food waste by half until 2030.

Among the most relevant issues that must be overcome is filling in the research gap regarding the drivers of food waste, from an integrated perspective, along the entire supply chain, as well as delivering information regarding what type of food is wasted in a specific segment of the supply chain. Food waste reduction measures must also rely on the interdependencies among different actors from the supply chain. Also, the analysis of everyday practices leading to food waste needs more attention. A relevant insight into the matter could support efficient information campaigns for the reduction of food waste.

Quantification efforts must be supported by using a robust methodology. By working together, researchers, public organizations and private companies can provide relevant information not only related to the total quantity of wasted food, but also related to the value of the food that is wasted and its carbon footprint. This way, the most efficient instruments and policies for food waste reduction can be identified.

Based on the results of the study, further research conducted by the author will focus on the analysis of the role that intelligent systems have on the reduction of food waste, as well as the effectiveness of online campaigns for the reduction of food waste among consumers.

\section{References}

Aschemann-Witzel, J., de Hooge, I.E., Rohm, H., Normann, A., Bonzanini Bossle, M., Grønhøj, A., Oostindjer, M. (2017). Key characteristics and success factors of supply chain initiatives tackling consumer-related food waste - A multiple case study. Journal of Cleaner Production, 155(Part 2), 33-45.

Beretta, C., Stoessel, F., Baier, U., Hellweg, S. (2013). Quantifying food losses and the potential for reduction in Switzerland. Waste Management, 33(3), 764-773.

Bratianu, C. (2015). Developing strategic thinking in business education. Management dynamics in the Knowledge Economy, 3(3), 409-429.

Bravi, L., Francioni, B., Murmura, F., Savelli, E. (2020). Factors affecting household food waste among young consumers and actions to prevent it. A comparison among UK, Spain and Italy, Resources, Conservation and Recycling, 153 (February 2020), Article No. 104586.

Bräutigam, K.R., Jörissen, J., Priefer, C. (2014). The extent of food waste generation across EU27: different calculation methods and the reliability of their results. Waste Management \& Research, 32(8), 683-694.

Buzby, J.C., Bentley, J.T., Padera, B., Ammon, C., Campuzano, J. (2015). Estimated Fresh Produce Shrink and Food Loss in U.S. Supermarkets. Agriculture, 5(3), 626-648.

Caldeira, C., De Laurentiis, V., Corrado, S., van Holsteijn, F., Sala, S. (2019). Quantification of food waste per product group along the food supply chain in the European Union: a mass flow analysis. Resources, Conservation and Recycling, 149 (October 2019), 479-488. 
Cicatiello, C., Franco, S. (2020). Disclosure and assessment of unrecorded food waste at retail stores. Journal of Retailing and Consumer Services, 52 (2020), Article No. 101932.

Cicatiello, C., Franco, S., Pancino, B., Blasi, E., (2016). The value of food waste: An exploratory study on retailing. Journal of Retailing and Consumer Services, 30(May 2016), 96-104.

Corrado, S., Sala, S. (2018). Food waste accounting along global and European food supply chains: State of the art and outlook. Waste Management, 79 (September 2018), 120-131.

Deloitte (2018). Artificial Intelligence Innovation Report. Retrieved from https://www2.deloitte.com/content/dam/Deloitte/de/Documents/Innovation/ArtificialIntelligence-Innovation-Report-2018-Deloitte.pdf

Elimelech, E., Ert, E., Ayalon, O. (2019). Bridging the gap between self-assessments and measured household food waste: A hybrid valuation approach. Waste Management, 95 (July 2019), 259-270.

Elimelech, E., Ayalon, O., Ert, E. (2018). What gets measured gets managed: A new method of measuring household food waste. Waste Management, 76 (June 2018), 68-81.

EPA, United States Environmental Protection Agency (2020). United States 2030 Food Loss and Waste Reduction Goal. Retrieved from https:/www.epa.gov/sustainable-managementfood/united-states-2030-food-loss-and-waste-reduction-goal

Eriksson, M., Malefors, C., Callewaert, P., Hartikainen, H., Pietiläinen, O., Strid, I. (2019). What gets measured gets managed - Or does it? Connection between food waste quantification and food waste reduction in the hospitality sector. Resources, Conservation \& Recycling: $X, 4$ (December 2019), Article No. 100021.

Eriksson, M., Persson Osowski, C., Björkman, J., Hansson, E, Malefors, C, Eriksson, E., Ghosh, R. (2018a). The tree structure - a general framework for food waste quantification in food services. Resources, Conservation and Recycling, 130 (March 2018), 140-151

Eriksson, M., Lindgren, S., Persson Osowski, C. (2018b). Mapping of food waste quantification methodologies in the food services of Swedish municipalities. Resources, Conservation and Recycling, 137 (October 2018), 191-199.

Eriksson, M., Strid, I., Hansson, P-A. (2012). Food losses in six Swedish retail stores: Wastage of fruit and vegetables in relation to quantities delivered. Resources, Conservation and Recycling, 68 (November 2012), 14-20.

European Commission (2020). EU Platform on food losses and food waste. Retrieved from https://audiovisual.ec.europa.eu/en/video/I-180488

EU Decision 2019/2000 (2019). Commission Implementing Decision (EU) 2019/2000 of 28 November 2019 laying down a format for reporting of data on food waste and for submission of the quality check report in accordance with Directive 2008/98/EC of the European Parliament and of the Council (notified under document C(2019) 8577)

Filimonau, V., Gherbin, A. (2017). An exploratory study of food waste management practices in the UK grocery retail sector. Journal of Cleaner Production, 167(November 2017), 11841194.

FLW Protocol (2016). Food Loss and Waste Accounting and Reporting Standard, FLW Protocol, Washington DC (2016)

Gallo, A.E. (1980). Consumer food waste in the United States. National Food Reviews, 12 (1980), 13-16.

Gokarn, S., Kuthambalayan, T.S. (2017). Analysis of challenges inhibiting the reduction of waste in food supply chain. Journal of Cleaner Production, 168(December 2017), 595-604. 
Graham-Rowe, E., Jessop, D.C., Sparks, P. (2015). Predicting household food waste reduction using an extended theory of planned behavior. Resources, Conservation and Recycling, 101(August 2015), 194-202.

Graham-Rowe, E., Jessop, D.C., Sparks, P. (2014). Identifying motivations and barriers to minimising household food waste. Resources, Conservation and Recycling, 84, 15-23.

Gustavsson, J., Cederberg, C., Sonesson, U. (2011). Global food losses and food waste: Extent, causes and prevention. Rome: Food and Agriculture Organization of the United Nations (FAO).

Hebrok, M., Heidenstrøm, N. (2019). Contextualising food waste prevention - Decisive moments within everyday practices. Journal of Cleaner Production, 210 (February 2019), 14351448 .

Ju, M., Osako, M., Harashina, S. (2017). Food loss rate in food supply chain using material flow analysis. Waste Management, 61(March 2017), 443-454.

Kor, Y.Y., Prabhu, J., Esposito, M. (2017). How Large Food Retailers Can Help Solve the Food Waste Crisis. Harvard Business Review, December 19, 2017. Retrieved from https://hbr.org/2017/12/how-large-food-retailers-can-help-solve-the-food-waste-crisis

Lee, K.C.L. (2018). Grocery shopping, food waste, and the retail landscape of cities: The case of Seoul. Journal of Cleaner Production, 172(January 2018), 325-334.

Leverenz, D., Moussawel, S., Maurer, C., Hafner, G., Schneider, F., Schmidt, T., Kranert, M. (2019). Quantifying the prevention potential of avoidable food waste in households using a self-reporting approach, Resources, Conservation and Recycling, 150 (November 2019), Article No. 104417.

Mena, C., Adenso-Diaz, B., Yurt, O. (2011). The causes of food waste in the supplier-retailer interface: Evidences from the UK and Spain. Resources, Conservation and Recycling, 55(6), April 2011, 648-658.

Bio Intelligence Service (2010). Final report - Preparatory study on food waste across EU 27; European Commission [DG ENV - Directorate C]. Paris: BIO Intelligence Service

Morone, P., Koutinas, A, Gathergood, N., Arshadi, M, Matharu, A. (2019). Food waste: Challenges and opportunities for enhancing the emerging bio-economy. Journal of Cleaner Production, 221 (June 2019), 10-16.

Özbük, R.M.Y, Coșkun, A. (2020). Factors affecting food waste at the downstream entities of the supply chain: A critical review. Journal of Cleaner Production, 244 (January 2020), Article No. 118628.

Papargyropoulou, E., Lozano, R., Steinberger, J.K., Wright, N., Bin Ujang, Z. (2014). The food waste hierarchy as a framework for the management of food surplus and food waste. Journal of Cleaner Production, 76(August 2014), 106-115.

Parfitt, J., Barthel, M., Macnaughton, S. (2010). Food waste within food supply chains: quantification and potential for change to 2050. Philosophical Transactions of the Royal Society B, 365(1554), 3065-3081.

Parizeau, K., von Massow, M., Martin, R. (2015). Household-level dynamics of food waste production and related beliefs, attitudes, and behaviours in Guelph, Ontario, Waste Management, 35 (2015), 207-217.

Priefer, C., Jörissen, J., Bräutigam, K.R. (2016). Food waste prevention in Europe - A causedriven approach to identify the most relevant leverage points for action. Resources, Conservation and Recycling, 109 (May-June 2016), 155-165. 
ReFED (2020). Stakeholders - Nonprofits \& Academia. Retrieved from https://www.refed.com/stakeholders/nonprofits-and-academia/

Romani, S., Grappi, S., Bagozzi, R.P., Barone, A.M. (2018). Domestic food practices: A study of food management behaviors and the role of food preparation planning in reducing waste, Appetite, 121(2018), 215-227.

Schanes, K., Dobernig, K., Gözet, B. (2018). Food waste matters - A systematic review of

PICBE $\mid 828$ household food waste practices and their policy implications. Journal of Cleaner Production, 182, 978-991.

Stefan, V., Van Herpen, E., Tudoran, A.A., Lähteenmäki, L. (2013). Avoiding food waste by Romanian consumers: the importance of planning and shopping routines. Food Quality and Preference, 28(1), 375-381.

Stenmarck, A., Jensen, C., Quested, T., Moates, G., Buksti, M., Cseh, B. et al. (2016). Estimates of European food waste levels. FUSIONS Report.

Teller, C., Holweg, C., Reiner, G., Kotzab, H. (2018). Retail store operations and food waste. Journal of Cleaner Production, 185 (June 2018), 981-997.

Van Boxstael, S., Devlieghere, F., Berkvens, D., Vermeulen, A., Uyttendaele, M. (2014). Understanding and attitude regarding the shelf life labels and dates on pre-packed food products by Belgian consumers. Food Control, 37(2014), 85-92.

Visschers, V.H.M., Wickli, N., Siegrist, M. (2016). Sorting out food waste behaviour: A survey on the motivators and barriers of self-reported amounts of food waste in households. Journal of Environmental Psychology, 45(March 2016), 66-78.

Williams, H., Wikström, F., Otterbring, T., Löfgren, M., Gustafsson, A. (2012). Reasons for household food waste with special attention to packaging, Journal of Cleaner Production, 24 (2012), 141-148.

Winnow Solution (2020). https://www.winnowsolutions.com/vision

WRAP, The Waste and Resources Action Programme (2020). UK progress against Courtauld 2025 targets and Sustainable Development Goal 12.3, Prepared by Andrew Parry, Billy Harris, Karen Fisher and Hamish Forbes, Banbury.

Xue, L., Liu, G., Parfitt, J., Liu, X., Van Herpen, E., Stenmarck, Å., O’Connor, C., Östergren, K., Cheng, S. (2017). Missing Food, Missing Data? A Critical Review of Global Food Losses and Food Waste Data. Environmental Science \& Technology, 51 (12), 6618-6633. 